\title{
Análisis ambiental del aluvión del año 2015 en la quebrada Rayo de Sol, Chosica, Lima, Perú
}

\author{
Environmental analysis of the alluvium of 2015 throughout the ravine \\ Rayo de Sol, Chosica, Lima, Peru
}

Nora Malca-Casavilca', Marcos Espinoza-Pereyra ${ }^{2}$

Recibido: Junio 2019 - Aprobado: Julio 2019

\begin{abstract}
RESUMEN
La presente investigación tuvo como objetivo realizar un análisis sobre la influencia de un evento natural (aluvión) ocurrido en marzo del 2015 en la quebrada Rayo de Sol3 - Chosica. Para llevar a cabo el análisis en la zona se emplearon cámaras fotográficas que sirvieron para registrar los daños desde la cuenca baja hasta la cuenca alta de la quebrada Rayo de Sol; un GPS etrex Garmin; una cinta métrica. La técnica fue la observación directa en el campo realizando el recorrido a pie. Se verificó que los ocho dispersores fueron totalmente destruidos por el aluvión, las viviendas en ambos cauces de la quebrada sufrieron daños considerables, así mismo la estructura de los servicios básicos; la escaza presencia o falta de vegetación nativa de la zona es evidente debido a la actividad antropogénica, y se evidenció capas de residuos sólidos urbanos en ambos cauces de la quebrada. El desconocimiento de los pobladores sobre el peligro de la zona donde decidieron ubicar sus viviendas así como las precipitaciones excepcionales y las malas prácticas en relación a desarrollo urbano han originado que el ambiente se vea afectado en los componentes tanto bióticos como abióticos.
\end{abstract}

Palabras clave: Análisis ambiental; quebrada; Rayo de Sol; Chosica; Aluvión.

\section{ABSTRACT}

The objective of this work was to perform an analysis on the influence of a natural event (alluvium) of March 2015 in the Rayo de Sol ravine. To carry out the analysis in the area, cameras were used to record the damage from the basin goes down to the upper basin of the Rayo de Sol ravine; a GPS etrex Garmin; a tape measure. The technique used was direct observation in the field by walking. It was verified that the eight dispersers were completely destroyed by the alluvium, the houses on both sides of the riverbed suffered considerable damage, as well as the structure of basic services; the scarcity presence or lack of native vegetation along the area is evident due to the anthropogenic activity, and urban solid waste layers were evidenced in both channels of the stream. The ignorance of the inhabitants about the danger of the area where they decided to locate their houses as well as the exceptional rainfall and bad practices in relation to urban development have caused the environment to be affected in both biotic and abiotic components.

Keywords: Environmental analysis; ravine; Rayo de Sol; Chosica; alluvium.

1 Docente de la UPG UNMSM, Magister en Ciencias Ambientales con mención en Control de la Contaminación y Ordenamiento Territorial. 2 Ingeniero Geógrafo de la UNMSM, Magister en Ciencias Ambientales con mención en Gestión y Ordenamiento Ambiental del Territorio. 3 También conocido como Rayos de Sol, Rayito de Sol o quebrada Corrales. 


\section{INTRODUCCIÓN}

De acuerdo con Strahler y Strahler (1987), el concepto de deslizamiento de tierras (movimientos en masa) es aplicado a todo movimiento de roca madre o regolito por una vertiente hacia altitudes bajas, esto es por efecto de la gravedad; los mismos autores recomienda el uso para volúmenes grandes que contengan rocas de gran tamaño.

El Grupo de Estándares para Movimientos en Masa (GEMMA, 2007) menciona que entre los tipos de movimientos en masa se tiene a la crecida de detritos que consiste en un flujo rápido de una crecida de agua y que transporta una gran cantidad de detritos a su paso. En el Perú son conocidos como aluviones o huaycos ${ }^{4}$.

La ocurrencia de aluviones en Chosica y Chaclacayo tienen una amplia documentación. Referencias de estos fenómenos se puede citar a los ocurridos en los años 1909, 1915, 1925, 1926, 1936, 1939, 1950,1952, 1954, 1955, 1959, 1967, 1972, 1976, 1983, 1985 y 1987, haciendo un énfasis en los años 1925-1926 ya que en conjunción con la aparición del Fenómeno del Niño, causaron estragos inclusive a la central hidroeléctrica de Huampaní (O'Connor, 1988); Carrillo y Guadalupe (2012) mencionan como catastrófico el ocurrido en el año de 1987.

El 23 de marzo del 2015 la quebrada Rayo de Sol sufrió daños como consecuencia de este tipo de deslizamientos (caracterizados por flujos de barro acompañados de rocas de diámetros variables y algún otro elemento que pueda arrastrar). Este tipo de fenómenos naturales son recurrentes, especialmente en la zona de Chosica. Los más afectados fueron los asentamientos humanos ubicados en los extremos laterales de la quebrada, esto ocurre como consecuencia de un evento lluvioso excepcional y el desarrollo de procesos erosivos provocados por los movimientos en masa en la parte alta y media de dicha quebrada y generando sedimentos, según el Instituto Nacional de Defensa Civil (INDECI). Se observó varias capas de residuos sólidos sobre las cuales se habían construido algunas viviendas, lo cual produjo inestabilidad y represamientos que al ser rebasados originaron las crecidas que causaron daños en las viviendas y servicios del distrito (ver figura 1).

De acuerdo con el Servicio Nacional de Meteorología e Hidrología del Perú (SENAMHI, 2015), y citado por Núñez, Villacorta y Huarez (2015), se sabe que la precipitación registrada en la estación de Chosica en el día 23 de marzo del 2015 se registro un pico de $18 \mathrm{~mm}$, pero los mismos autores acotan que existen registros de precipitación que datan desde el 17 de marzo del 2015 incrementándose hasta llegar el día del evento (23 de marzo del 2015) especulando que la lluvia se acumulo entre esas fechas hasta saturar las quebradas de la zona (ver figura 2).

Se plantea la hipótesis que pudieran ocurrir otros eventos, como los aluviones, en la quebrada debido a la ocurrencia de lluvias torrenciales en la zona, posiblemente incrementadas en volumen debido al cambio climático que afecta al planeta Tierra, ya que el Perú según el Tyndall Center de Inglaterra como lo cita el Ministerio de Ambiente

\footnotetext{
4 La palabra "huayco" proviene de la palabra quechua "wayqu" que significa quebrada.
}

del Perú (MINAM, 2015), es el tercer país más vulnerable al cambio climático, siendo la vulnerabilidad climática el grado de susceptibilidad de un espacio natural o territorio que puede variar a la exposición, sensibilidad y capacidad adaptativa a un cambio.

Debido al aluvión se produjo el transporte, por el cauce de la quebrada, de materiales expuestos en la superficie como troncos, piedras, residuos maderables, residuos sólidos urbanos, sedimentos, entre otros. Estos materiales llegaron al Jirón Jorge Chávez y se obstruyó en las viviendas del barrio Buenos Aires, lo que ocasionó la interrupción del tráfico normal a la altura del kilómetro 36 de la carretera central, que produjo desabastecimiento a la capital.

Es importante recalcar que los aluviones causan grandes daños en la infraestructura como casas, redes de telefonía, electricidad, de agua y desagüe, como también las vías de transporte (pistas vecinales y carreteras) provocando bloqueos en las ciudades y en consecuencia se corta las provisiones llegando a generar grandes pérdidas económicas al país y también es un peligro para la vida de los pobladores que habitan en lugares donde usualmente ocurren estos eventos.

\section{METODO}

Para el análisis de la situación se contó con la participación de la coordinadora, los y las estudiantes de la facultad de ingeniería ambiental de la Universidad Privada del Norte, un profesional ingeniero geógrafo de la Universidad Nacional Mayor de San Marcos, así como una de las lideresas de la zona la Sra. Elida de quién tuvimos la oportunidad de escuchar el relato de lo sucedido el 23 de marzo y las consecuencias de la fuerte precipitación, los rayos y truenos que se sucedieron éste día.

\subsection{Descripción general de la micro cuenca Rayo de Sol}

La unidad de análisis es una de las quebradas de la zona denominada "Rayo de Sol" (Figura 3), ubicada en la margen derecha del río Rímac, teniendo una longitud de $1.0 \mathrm{~km}$, e inicia a 1,450 m.s.n.m., con un rumbo $\mathrm{N} 40^{\circ} \mathrm{W}$. Posee fuerte pendiente de $40 \%$ (Figura 4), en otras publicaciones se le considera como una cárcava. La micro cuenca de la quebrada Rayo de Sol tiene un ancho de $14 \mathrm{~m}$ aproximadamente en su zona central y la cuenca tiene 500 $\mathrm{m}$, que da una superficie colectora de $7000 \mathrm{~m}^{2}$.

Litológicamente en el área de estudio tenemos un afloramiento de rocas intrusivas del batolito de la costa (Palacios, Caldas y Vela, 1992), como Súper Unidad Patap, son rocas con una datación entre 84 - 102 millones de años atrás (Pitcher, 1985). Estas rocas se encuentran ubicada en la parte final de la quebrada Cantuta y alrededores. Otro grupo es la Súper Unidad Santa Rosa, que se encuentra presente en la mayoría de los cerros y quebradas de Chosica. La Super Unidad Santa Rosa está dividida en dos subunidades: subunidad Tonalita - Dioritas (Santa Rosa Oscuro), que aflora mayormente en los cerros de La Cantuta; y subunidad Tonalita - Granodioritas (Santa Rosa Claro). También se encuentran depósitos cuaternarios, que incluye depósitos de las terrazas del río Rímac, depósitos de huayco 


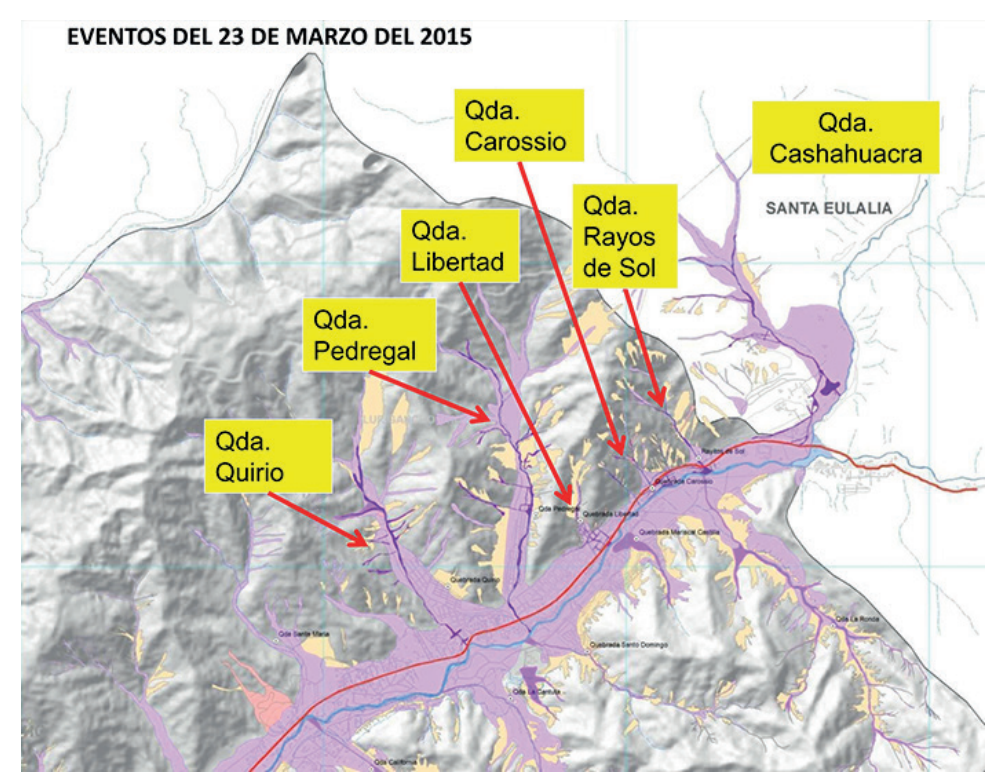

Figura 1. Principales Quebradas de Chosica.

Fuente. Núñez, Villacorta, Huarez y Fidel, 2015

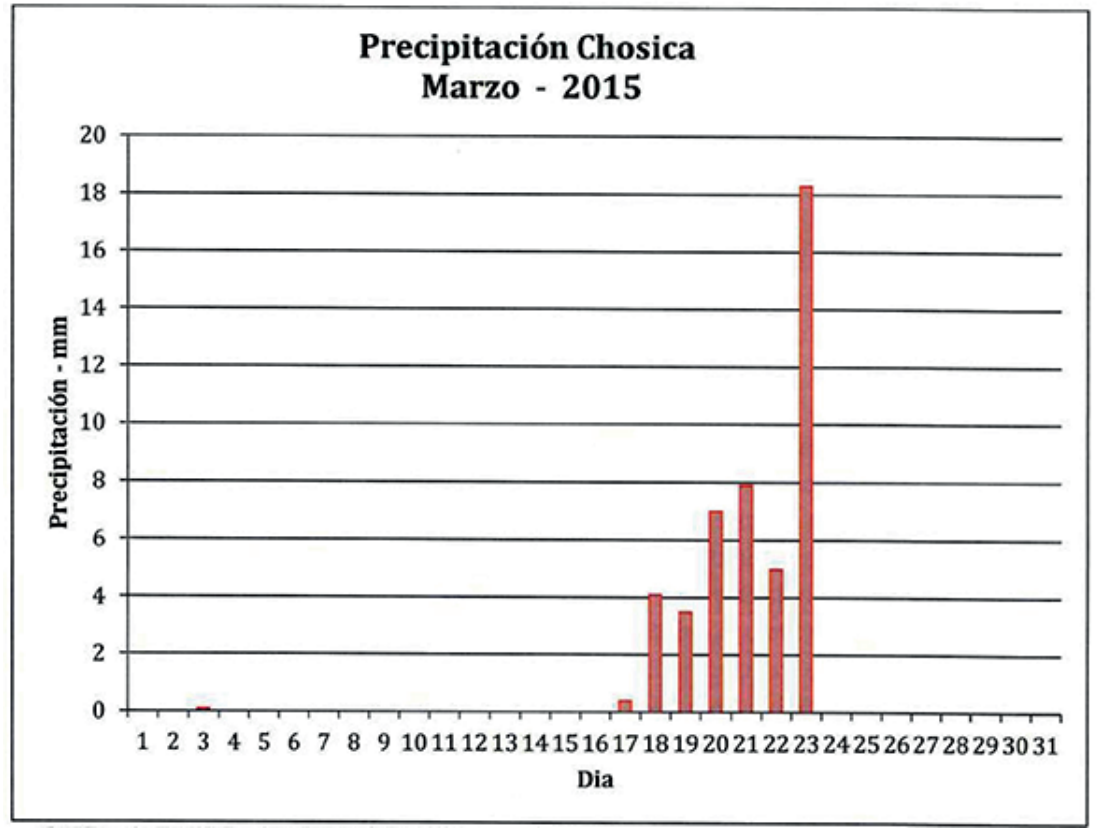

Figura 2. Precipitaciones pluviales registradas en la estación Chosica en el mes de marzo del 2015. Fuente. Tomado de Núñez, Villacorta y Huarez (2015) con datos del SENAMHI (2015).

encontrados en las quebradas y los depósitos coluviales (formados por gravedad) ubicados al pie de algunos cerros. Cabe señalar que la mayoría de los asentamientos humanos se encuentran emplazados en depósitos aluvionales formados por la disgregación las rocas de la Súper Unidad Santa Rosa. (Carrillo y Guadalupe, 2012). Los procesos geológico-climáticos que ocurren en forma recurrente son: huaycos (aluviones) y desprendimiento de rocas de acuerdo con lo mencionado por el Instituto Nacional de Defensa Civil (INDECI, 2005) (Figura 5).

De acuerdo con Palacios, Caldas y Vela (1992) el área de la quebrada Rayo de Sol esta designada con una geoforma denominada Estribaciones Andinas Occidentales la que le corresponde una topografía rugosa, con plutones y stocks del Batolito de la Costa disectados por el rio Rímac y las quebradas tributarias, laderas y crestas marginales de la cordillera. A una mayor escala de detalle se subdivide en tres geoformas: Flanco Occidental de los Andes, Quebradas Depósitos de Huaycos, y Terrazas Fluviales (Figura 6).

\subsection{Descripción de la situación antes del evento del 23 de marzo del 2015}

En la quebrada Rayo de Sol (y como es en general en las quebradas aledañas) es común la presencia de ocho dispersores de energía, que tienen como misión la de 
reducir la velocidad de agua y retener las rocas de gran tamaño (Gallarday y Malca, 2014), y dispuestos en forma perpendicular al eje de la quebrada, como también sacos de arena en el frontis de las casas ubicadas en la parte media y baja de la quebrada y sobrepuestos entre ellos, estos sacos de arena también se suelen ver en el sector sur de la Carretera Central. Existía presencia de pocos árboles en los extremos de la quebrada. La población se ha asentado de forma desorganizada en el asentamiento humano del jirón Jorge Chávez ubicado en el eje de la quebrada (Figura 7).

\section{RESULTADOS}

En el trabajo de campo realizado, se constató la acumulación de piedras de gran tamaño y heterogéneo, la presencia de vegetación en ambos extremos laterales es escasa, y la pendiente es empinada mientras que los ocho dispersores de energía fueron destruidos. El aluvión paso por el jirón Jorge Chávez que sumado a la fuerte pendiente llega hasta la Carretera Central. Existe la presencia de un relleno de residuos domésticos compactados en los lados de la quebrada haciendo inestable el suelo y paredes de la quebrada.

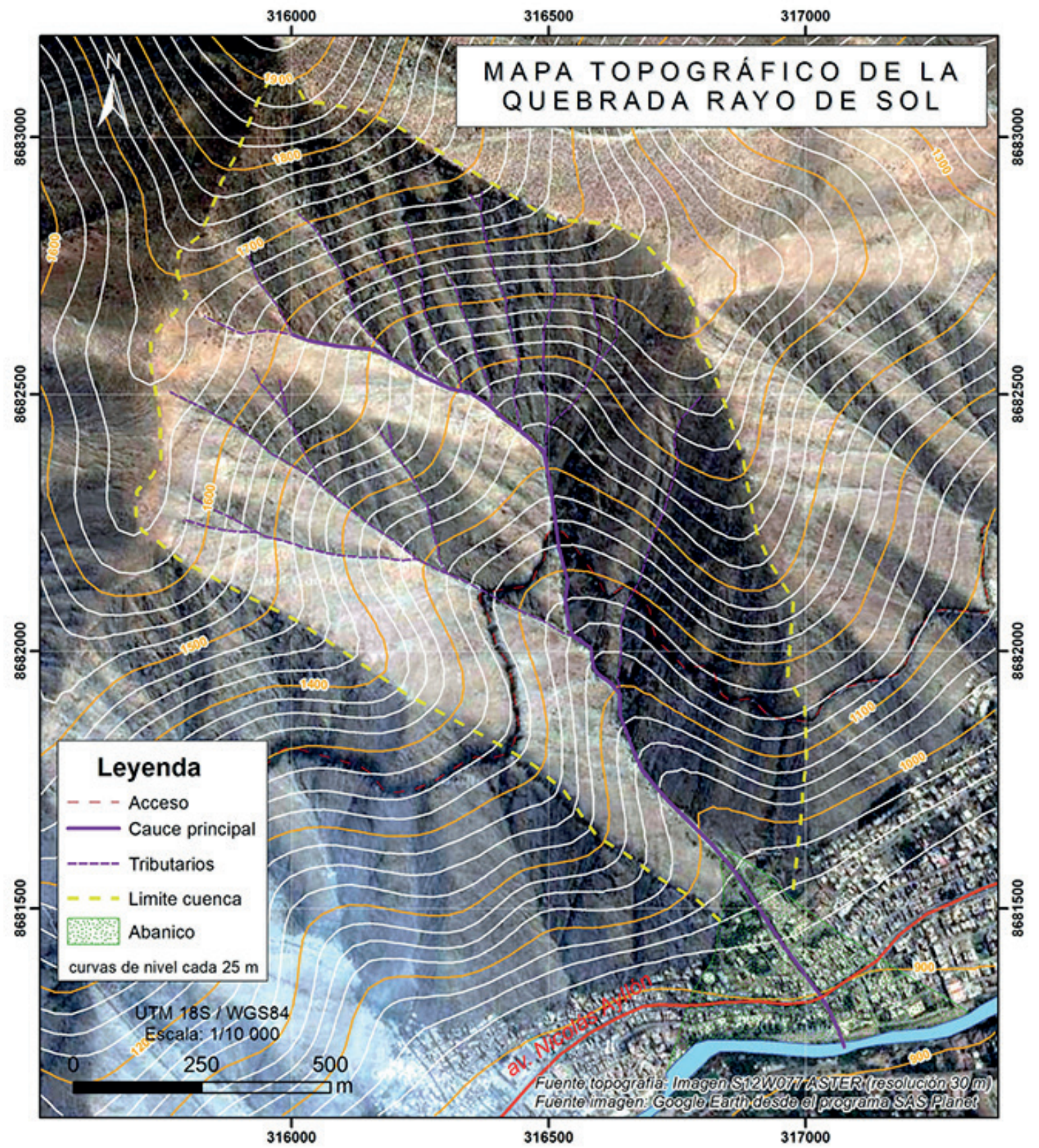

Figura 3. Mapa topográfico de la cuenca Rayo de Sol.

Fuente. Elaboración propia en base a una imagen de satélite proveniente del programa Google Earth y las curvas de nivel provenientes de una imagen Aster del geoservidor del Ministerio del Ambiente del Perú (MINAN, 2019) con resolución espacial cada $30 \mathrm{~m}$ 


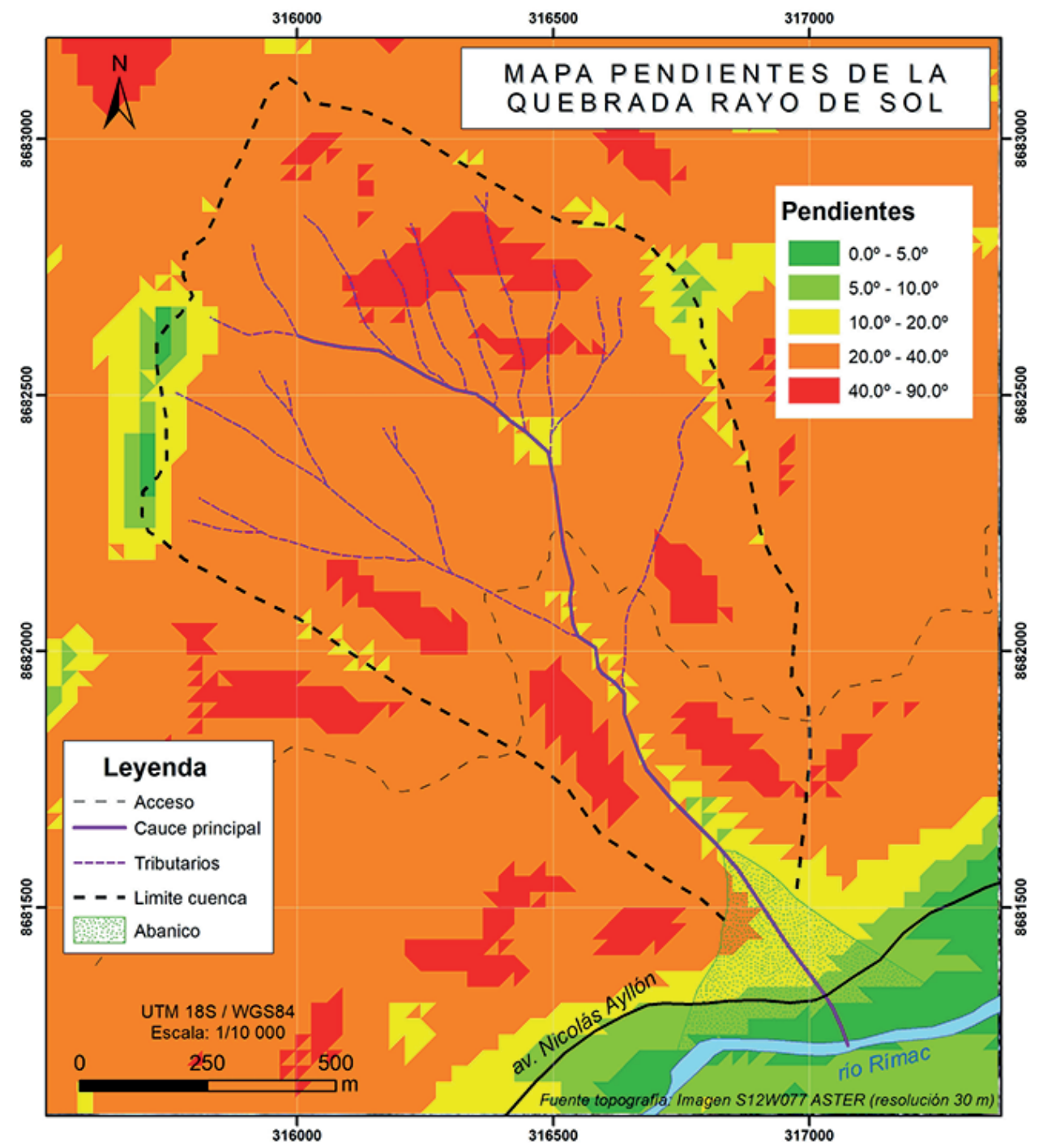

Figura 4. Mapa topográfico de la cuenca Rayo de Sol.

Fuente. Elaboración propia en base a una imagen de satélite proveniente del programa Google Earth y las curvas de nivel provenientes de una imagen Aster del geoservidor del Ministerio del Ambiente del Perú (MINAN, 2019) con resolución espacial cada $30 \mathrm{~m}$.

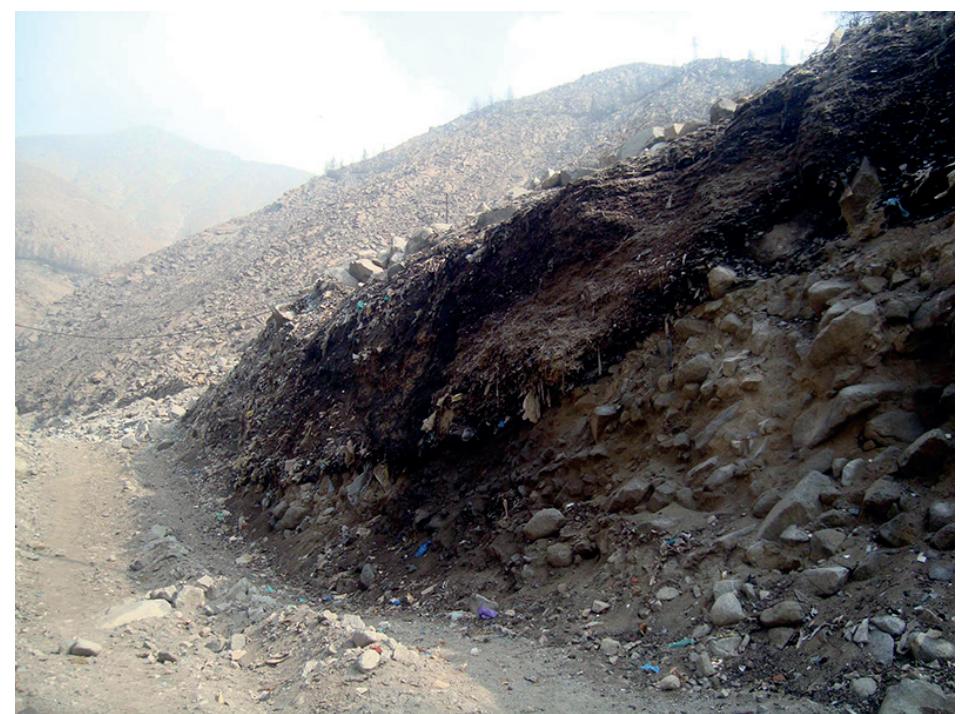

Figura 5. Se observa una capa de residuos sólidos urbanos en el cauce de la quebrada. Fuente. Elaboración propia 


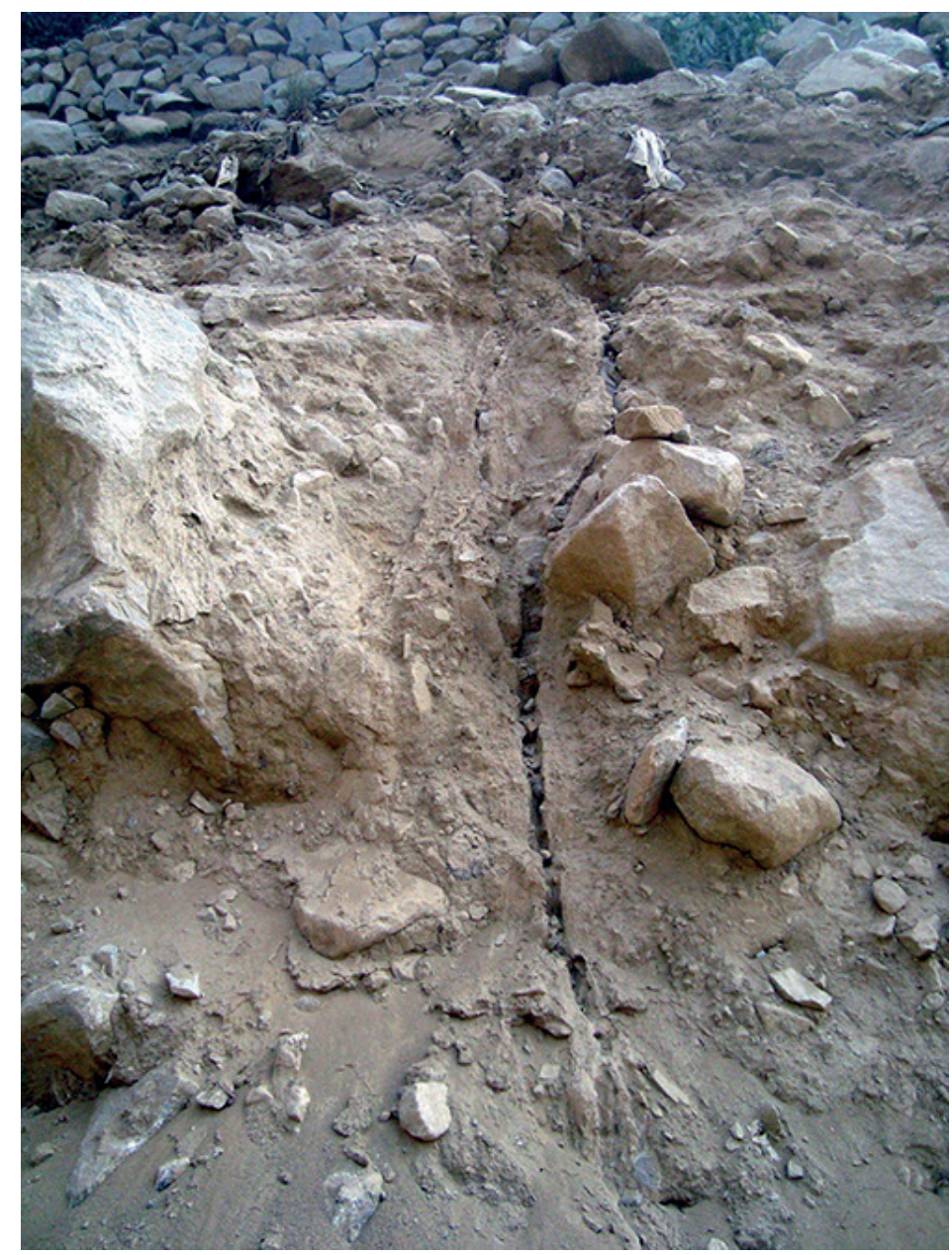

Figura 6. Material aluvial (rocas provenientes de las partes altas de la quebrada y sedimentos). Se observa también una fractura. Fuente. Elaboración propia

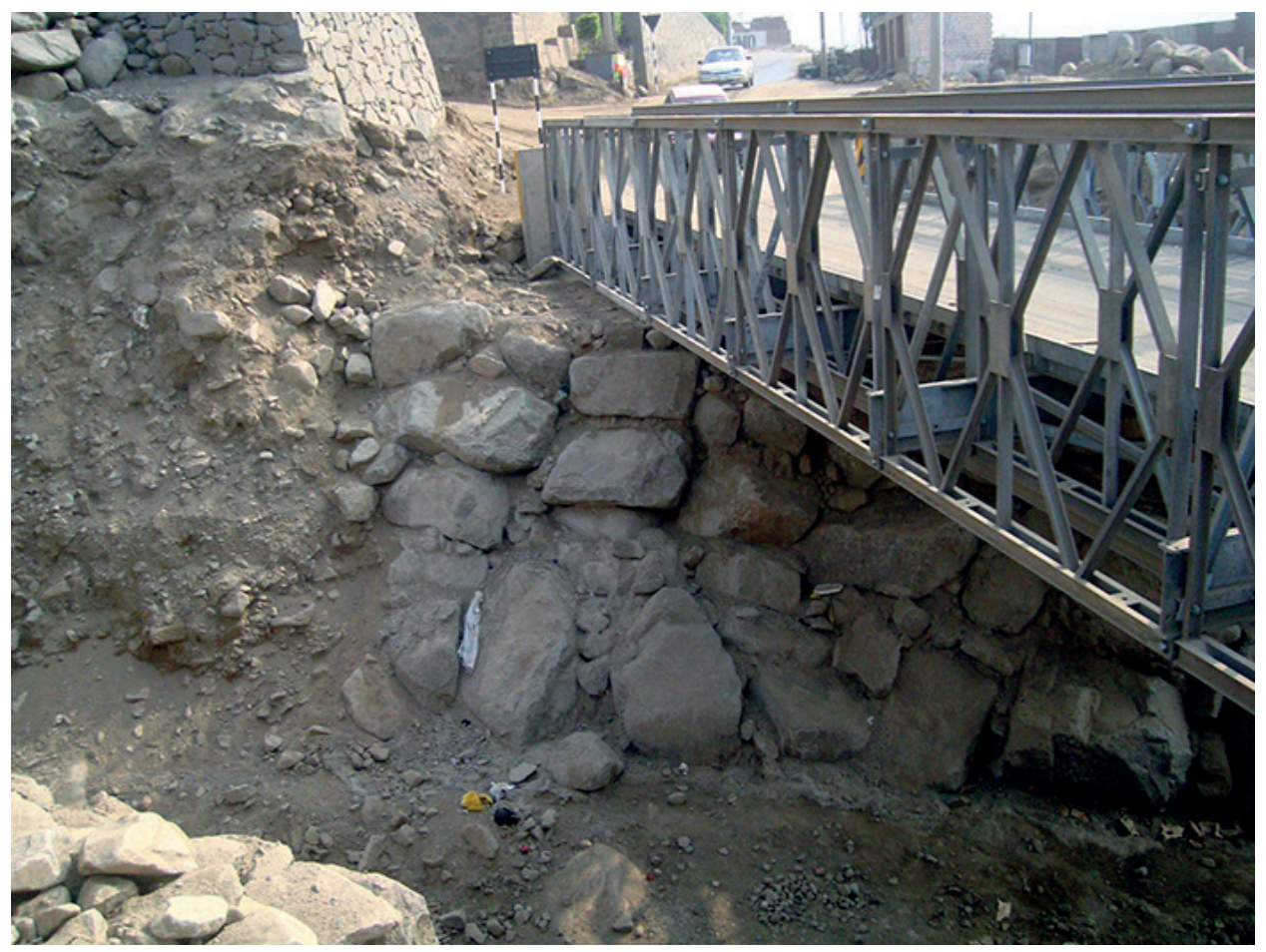

Figura 7. Puente para el paso de vehículos y personas en la parte baja de la quebrada (las bases son de rocas de gran diámetro). Fuente. Elaboración propia 


\section{DISCUSIÓN}

En esta pequeña micro cuenca se observa el problema común en la zona de Chosica: en el querer habitar en zonas con una geodinámica muy dinámica. Los ocho disparadores al final no cumplieron su función ya que al final el aluvión llego a la Carretera Central, por lo tanto, se puede inferir que no hubo un buen calculo de caudal (incluso los pobladores afirmaban que nunca habían visto un evento de esta forma). Existe una malla que tiene como fin la contención de grandes bloques de roca provenientes de la parte, pero no protege de los bloques en la parte media (tal vez estos fueron los que destruyeron los ocho disparadores). También se puede inferir que hubo arrastre de residuos sólidos existentes en los lados de la quebrada (y que de por si esto es un foco infeccioso).

La pendiente también ayudo a que las rocas y general el flujo aluvional golpe con fuerza y destruya las infraestructuras destinadas a la contención de estos mencionados, se infiere, que no fue tomado en cuenta al hacer el diseño final.

\section{CONCLUSIONES}

Luego del análisis y de la discusión, se tiene estas dos conclusiones:

- En insistir que estas zonas no son aptas para la vivienda, si bien estos eventos de estas magnitudes no suelen suceder a cada momento, al final es mejor evitar algún percance y la perdida de vidas e infraestructuras.

- El no tener la visión de un sistema integral a nivel de cuenca de protección o contención ante este tipo de eventos ya que se evidencia el mal (o nulo) cálculo del caudal máximo y el tiempo de retorno, y sumar la influencia de la pendiente, y en conjunto diseñar una buena red de contención en la parte alta, arborización en los lados de la quebrada, también la remoción de los residuos domésticos encontrados y el rediseño de los disipadores de energía con un programa de descolmatación del eje de la quebrada.

\section{RECOMENDACIONES}

- Brindar capacitaciones y simulacros a los pobladores de las quebradas afectadas por estos eventos recurrentes.

- Como medida de prevención, programar el plantado de árboles en la parte alta de la quebrada para mitigar los efectos de la lluvia.

- Realizar estudios de suelos para determinar la estabilidad de los mismos con fines preventivos como también estudios de zonificación ambiental para la determinación de la mejor vocación de los suelos (para fines de vivienda, comercial, agrario, turismo, entre otros).
- Reconstruir diques en las nacientes de las principales quebradas como el mantenimiento frecuente y la canalización de los mismos.

- Realizar programas de segregación y disposición final de los residuos sólidos urbanos como la remoción de obstáculos de las quebradas afluentes del rio Rímac.

- Prohibir la construcción de viviendas en zonas de alto riesgo por movimientos de masa. Esto es posible con ordenanzas a nivel distrital o provincial.

\section{REFERENCIAS BIBLIOGRAFICAS}

Carrillo, N. y Guadalupe, E. (2012). Caracterización y análisis de los huaycos del 5 de abril del 2012 Chosica- Lima. Rev. del Instituto de Investigación (RIIGEO), FIGMMG-UNMSM Vol. 15, N. ${ }^{\circ}$ 29, pp. 69- Enero - Junio 2012. Recuperado de http://hdl.handle.net/123456789/2808

Gallarday, T y Malca, N. (2014) Flujos de detritos y dispersores de energía en la torrentera Juan Carossio Chosica - Lima. Rev. del Instituto de Investigación (RIIGEO), FIGMMGUNMSM Vol. 17, N. ${ }^{\circ}$ 33, pp. 39-44. Enero -Junio 2014. Recuperado de https://revistasinvestigacion.unmsm.edu.pe/ index.php/iigeo/article/view/11495

Grupo de Estándares para Movimientos en Masa (GEMMA, 2007). Movimientos en masa en la región andina: una guía para la evaluación de amenazas. Publicación Geológica Multinacional No4, 2007. Proyecto Multinacional Andino: Geociencias para las Comunidades Andinas. Publicación Geológica Multinacional, No. 4, 432 p. Recuperado de http://repositorio.segemar.gov.ar/308849217/2792

Instituto Nacional de Defensa Civil (INDECI, 2005). Proyecto INDECI - PNUD PER/02/051. Mapa de peligros y plan de usos del suelo y medidas de mitigación ante desastres de la ciudad de Chosica. Resumen Ejecutivo. Ciudades Sostenibles-Programa de las Naciones Unidas para el Desarrollo-PNUD. 48 pág. Recuperado de http://bvpad. indeci.gob.pe/doc/estudios CS/Region_Lima/lima/ chosica_R.pdf

Ministerio del Ambiente del Perú (MINAM, 2015). La Libertad: ¿Por qué el Perú es el tercer país más vulnerable al cambio climático?. Recuperado de https://sinia.minam.gob.pe/ contenido/libertad-porque-peru-tercer-pais-mas-vulnerablecambio-climatico

Ministerio del Ambiente del Perú (MINAM, 2019). Geoservidor - información ASTER GDEM. Recuperado de http:// geoservidorperu.minam.gob.pe/geoservidor/download raster.aspx

Núñez Juárez, S., Villacorta Chambi, S. y Huarez Yarleque, C. (2015). Evaluación geodinámica de los detritos del 23/03/20/2015 entre las quebradas Rayos de Sol y Quirio (Chosica) y Cashahuacra (Santa Eulalia). Recuperado de https://hdl.handle.net/20.500.12544/1310

O’Connor Salmón, H. (1988). Investigación del Huayco de Chosica 1987, sus efectos y medidas de mitigación.; Lima: Universidad Nacional de Ingeniería. Tesis para optar el título profesional de Ingeniero Civil. 99 pp 
Palacios O., Caldas J. y Vela Ch. (1992) Geología de los Cuadrángulos de Lima, Lurín, Chancay y Chosica. Boletín $\mathrm{N}^{\circ}$ 43, INGEMMET Serie A, 163p. Recuperado de https:// hdl.handle.net/20.500.12544/163

Pitcher, W. (1985) A multiple and composite batholith. En: Pitcher, W.S., et al. (eds.), Magmatism at a plate edge: the Peruvian Andes, John Wiley, New York, pp. 93-107. https:// doi.org/10.1007/978-1-4899-5820-4_9

Servicio Nacional de Meteorología e Hidrología del Perú (SENAMHI, 2015). Datos de precipitación de la estación Chosica del 23/03/20/2015. Recuperado de https://www. senamhi.gob.pe/?p=aviso-hidrologico-detalle $\& \mathrm{a}=2015 \& \mathrm{~b}=$ $006 \& \mathrm{c}=026 \& \mathrm{~d}=$ SENA
Strahler, A. y Strahler A. (1987). Geografía Física [Traducido al español de Modern Physical Geography]. Barcelona: Ediciones Omega. Recuperado de https://geografiafisica. org/sem201901/geo112/bibliografia/articulos_libros/ geografiafisica/209850219-00-Portada-IntroduccionGEOGRAFIA-FISICA-STRAHLER.pdf 